\title{
Awake prone positioning in non-intubated patients for the management of hypoxemia in COVID-19: A systematic review and meta-analysis
}

\author{
Sryma PB ${ }^{1 *}$, Saurabh Mittal ${ }^{1 *}$, Karan Madan ${ }^{1}$, Anant Mohan ${ }^{1}$, Pawan Tiwari ${ }^{1}$, Vijay Hadda1, \\ Ravindra Mohan Pandey ${ }^{2}$, Randeep Guleria ${ }^{1}$ \\ ${ }^{1}$ Department of Pulmonary, Critical Care and Sleep Medicine; ${ }^{2}$ Department of Biostatistics, All India Institute of Medical \\ Sciences (AIIMS), New Delhi, India
}

*These authors contributed equally and are the joint first authors of this manuscript

\begin{abstract}
Coronavirus disease-2019 (COVID-19) may lead to hypoxemia, requiring intensive care in many patients. Awake prone positioning (PP) is reported to improve oxygenation and is a relatively safe modality. We performed a systematic review of the literature to evaluate the available evidence and performed meta-analysis of the effect of awake PP in non-intubated patients on improvement in oxygenation and reducing the need for intubation. We searched the PubMed and EMBASE databases to identify studies using awake PP as a therapeutic strategy in the management of COVID-
\end{abstract}

Correspondence: Dr. Saurabh Mittal, Assistant Professor, Department of Pulmonary, Critical Care and Sleep Medicine, All India Institute of Medical Sciences (AIIMS), Ansari Nagar, New Delhi 110029, India. Tel. +91.11.26546346.

E-mail: saurabh_kgmu@yahoo.co.in

Authors' contributions: SPB, data extraction and manuscript preparation; SM, study concept, studies search, data extraction, statistical analysis, manuscript writing; KM, data extraction, manuscript revision; AM, PT, VH, RG, manuscript revision; RMP, statistical analysis. All the authors have read and approved the final version of the manuscript and agreed to be accountable for all aspects of the work.

Availability of data and materials: The data used to support the findings of this study are available from the corresponding author on reasonable request.

Conflict of interest: The authors declare that they have no competing interests, and all authors confirm accuracy.

Key words: Awake proning; COVID-19; prone positioning; SARSCoV-2.

Received for publication: 26 September 2020.

Accepted for publication: 28 January 2021.

${ }^{\circ}$ Copyright: the Author(s), 2021

Licensee PAGEPress, Italy

Monaldi Archives for Chest Disease 2021; 91:1623

doi: 10.4081/monaldi.2021.1623

This article is distributed under the terms of the Creative Commons Attribution Noncommercial License (by-nc 4.0) which permits any noncommercial use, distribution, and reproduction in any medium, provided the original author(s) and source are credited.
19. Studies were included if they reported respiratory outcomes and included five or more subjects. The quality of individual studies was assessed by the Qualsyst tool. A meta-analysis was performed to estimate the proportion of patients requiring intubation. The degree of improvement in oxygenation parameters $\left(\mathrm{PaO}_{2}\right.$ : $\mathrm{FiO}_{2}$ or $\mathrm{PaO}_{2}$ or $\mathrm{SpO}_{2}$ ) was also calculated. Sixteen studies (seven prospective trials, three before-after studies, six retrospective series) were selected for review. The pooled proportion of patients who required mechanical ventilation was 0.25 (95\% confidence interval (CI) 0.16-0.34). There was a significant improvement in $\mathrm{PaO}_{2}: \mathrm{FiO}_{2}$ ratio, $\mathrm{PaO}_{2}$, and $\mathrm{SpO}_{2}$ during awake PP. To conclude, there is limited evidence to support the efficacy of awake PP for the management of hypoxemia in COVID-19. Further RCTs are required to study the impact of awake PP on key parameters like avoidance of mechanical ventilation, length of stay, and mortality.

\section{Introduction}

In December 2019, a novel coronavirus (severe acute respiratory syndrome coronavirus 2; SARS-CoV-2) emerged in China and spread globally, creating a pandemic. The disease results in a significant number of critically ill patients with the requirement of intensive unit care (ICU) admission and invasive mechanical ventilation (IMV) [1]. Among patients who need IMV, reported mortality is high varying from $49 \%-88 \%[1,2]$. Among hospitalized patients of COVID-19, the incidence of acute respiratory distress syndrome (ARDS) is reported to be about 33\% and sometimes as high as $68 \%$ [3]. In intubated patients with moderate to severe ARDS, prolonged prone positioning has been shown to improve oxygenation, and reduce mortality [4]. Prone positioning (PP) improves oxygenation by multiple mechanisms, such as redistribution of blood flow and edema fluid to the ventral side with gravity and reopening of atelectatic alveoli, which causes improvement in ventilation-perfusion mismatch $[5,6]$. However, PP requires the initiation of deep sedation as well as neuromuscular blocking agents and may be associated with complications in the form of obstruction and displacement of the endotracheal tube (ET) or venous catheter [5]. Awake PP may have similar advantages in improving oxygenation and possibly reduce the need for IMV without the associated problems of deeper sedation and ET displacement [7]. Before the pandemic, awake PP was used sparingly and has shown to improve oxygenation and reduce intubation rates in hypoxic respiratory failure $[8,9]$. An intervention that may reduce mortality, especially one that can be easily implemented at little additional cost, requires adequate data to support its 
benefits and possible harms. There is emerging data on the use of awake prone positioning to manage COVID-19 related hypoxemic respiratory failure $[10,11]$. This systematic review and meta-analysis aim to summarize the current evidence of awake PP in COVID19 hypoxemic respiratory failure in non-intubated patients.

\section{Methods}

The report of this systematic review was made according to the recommendations of the Preferred Reporting Items for Systematic Reviews and Meta-Analyses (PRISMA) statement [12].

\section{Eligibility criteria}

We included studies on adults (at least five patients) with COVID-19 and hypoxemic respiratory failure, not requiring IMV, and employing awake PP as a therapeutic strategy. We excluded small patient series (less than five) as they are highly likely to present a biased outcome in the form of only favorable outcome reporting [13]. We excluded studies on PP during IMV as well as studies not reporting respiratory outcomes. There was no comparator group.

\section{Search strategy and initial review}

A systematic search was performed in the PubMed and EMBASE databases to look for studies concerning awake PP in COVID-19 till July 26 ${ }^{\text {th }}, 2020$. The following search terms were used: ("prone" OR "proning" OR "prone positioning") AND ("COVID-19" OR "SARS CoV-2" OR “ SARS CoV 2"). Only English language studies were included. All the retrieved citations were imported into reference management software (EndNote) by two authors independently (SM and SP). The duplicate references were removed, and all references were screened through titles and abstracts. The reference lists of the extracted studies were also reviewed to look for relevant articles.

\section{Study selection}

Two review authors (SM and SP) independently screened and classified all citations as potential case-control studies, review articles, case series, or others for inclusion. We included all prospective, retrospective as well as randomized controlled studies reporting respiratory outcomes following awake PP in COVID-19 related hypoxemic respiratory failure. We defined COVID-19 hypoxemic respiratory failure as patients with confirmed COVID-19 infection requiring oxygen supplementation or with room air saturation less than $94 \%$. Awake PP was defined as usage of prone positioning in a conscious alert patient, not on IMV, irrespective of the duration of proning. The primary outcome was the need for endotracheal intubation and IMV. Other outcomes included indices of oxygenation, mortality, and length of stay.

Two review authors (SM and SP) examined all potential studies and decided whether they should be included in the review. Any disagreement was resolved by further discussion with a third author (KM).
Articles identified by the search were assessed for suitability. The primary outcome analyzed was the need for IMV. Other secondary outcomes included change in oxygenation status as assessed by pulse oxygen saturation $\left(\mathrm{SpO}_{2}\right)$, pulse oxygen saturation and the fraction of inspired oxygen ratio $\left(\mathrm{SpO}_{2}: \mathrm{FiO}_{2}\right)$, the partial pressure of oxygen and fraction of inspired oxygen ratio $\left(\mathrm{PaO}_{2}\right.$ : $\mathrm{FiO}_{2}$ ), respiratory rate, mortality, length of stay, and adverse events of awake PP. In the case of non-intubated patients, the expected concerns with prone positioning include worsening of respiratory failure due to non-tolerance, pressure sores, back pain, vomiting, and issues of venous access.

\section{Data abstraction}

Two review authors (SM and SP) extracted and reviewed the data. Data from the finally selected studies were extracted on a data extraction form. By thorough review of the article, the following information was retrieved - (a) author, (b) year, (c) number of patients, (d) country, (e) inclusion criteria, (f) study design, (e) age, (f) gender, (g) intervention, (h) outcome measures including the number of patients requiring intubation, pre- and post-intervention oxygenation indices and respiratory rate, mortality, length of stay (i) strengths, and (j) limitations of the study.

Data, if not reported as mean and standard deviation, were derived from individual patient data given in the original papers or supplementary data using Stata software. In one study with a control group, we included data only from the group undergoing awake PP [6].

\section{Assessment of study quality}

The Qualsyst tool for quantitative studies was used to assess the quality of studies $[14,15]$. Two authors (S.M. and S.P.B.) evaluated the quality of the selected studies for meta-analysis. The definition of the quality of a paper was defined as: strong (summary score of $>0.80$ ), good (summary score of $0.71-0.79$ ), adequate (summary score of $0.50-0.70$ ), and limited (summary score of $<0.50)$ [14].

\section{Statistical analysis}

Statistical analyses were performed using the STATA statistical analysis software (StataCorp. 2017. Stata Statistical Software: Release 15. StataCorp LLC., College Station, TX, USA) The Proportional meta-analysis was performed using the randomeffects model for the primary outcome (i.e, the need for intubation). This data was extracted from the studies as the number of patients having the outcome of interest divided by the total number of patients. The forest plots were generated using Stata software for proportional meta-analysis.

When the same measure of oxygenation was reported in studies, the pooled effect of change in each oxygenation parameter $\left(\mathrm{SpO}_{2}, \mathrm{PaO}_{2}, \mathrm{PaO}_{2}\right.$ : FiO2) and respiratory rate were presented as a weighted mean difference with corresponding $95 \%$ confidence intervals. The analyses for these outcomes were conducted using the means and standard deviations provided in the articles. These forest plots were generated using Revman 5 software. When dif- 
ferent measures of oxygenation such as $\mathrm{PaO}_{2}$ : FiO $2, \mathrm{PaO}_{2}, \mathrm{SpO}_{2}$ were reported, we estimated the pooled effect using standardized mean difference (SMD) with $95 \%$ confidence intervals by inverse variance statistical method. If a study reported more than one oxygenation parameter, we preferred $\mathrm{PaO}_{2}$ : $\mathrm{FiO}_{2}$ and $\mathrm{PaO}_{2}$ change over $\mathrm{SpO}_{2}$ for the estimation of SMD. A qualitative synthesis of data was performed in case the data for meta-analysis was not available.

\section{Heterogeneity and publication bias assessment}

The impact of heterogeneity on the pooled estimates of the outcome was assessed using the Cochran Q statistic and $\mathrm{I}^{2}$ test (measures the extent of inconsistency among the results of the studies) [16]. The presence of publication bias assessment was done using the funnel plot, which is a measure of the proportion (in the X-axis) against the standard error of the proportion (in the Y-axis). The minimum number of studies required for a funnel plot is usually 10 and Begg's test was used for publication bias assessment.

\section{Results}

The search yielded 221 citations out of which we accessed 57 full-text articles (Figure 1). A total of 16 articles (six prospective cohort studies [6,11,17-20], four before-after studies [10,21-23], and six retrospective cohort studies [24-29]) including a cumula- tive 316 patients with COVID-19 acute hypoxic respiratory failure undergoing awake PP were included for final review. No randomized controlled trials or systematic reviews were available.

\section{Study characteristics}

The basic details of the 16 included studies are summarized in Table 1. Female patients comprised $32.1 \%$ of the total population (89/ 277 patients, 14 studies). The interfaces used for oxygen therapy varied from conventional oxygen therapy with nasal cannula, face mask or non-rebreather mask $[6,11,17,18,22,26,27,29]$, high flow nasal cannula $[11,20,26,28,29]$, continuous airway positive pressure (CPAP) [17,19,25], and non-invasive ventilation (NIV) [10,21] including helmet NIV/ CPAP [19,21]. The same study had applied multiple methods of oxygen delivery as per the requirement of the patients. Among the 262 patients for whom interface data was available, $108(41.2 \%)$ were on positive airway pressure therapy by CPAP or NIV. The severity of hypoxia in the included studies indicates a moderate ARDS, with a mean $\mathrm{PaO}_{2}: \mathrm{FiO}_{2}$ ratio of 161.7 from seven studies that reported the $\mathrm{PaO} 2$ : FiO2 ratio before awake PP. The proning protocol used in studies also varied widely, with studies reporting the mean duration of awake PP per day from 2 hours to 9 hours [20,21]. In another study, $63 \%$ of the patients enrolled were able to continue awake PP for more than 3 hours per day [17]. One study also employed lateral positioning depending upon the radiological distribution of infiltrates, with prone positioning used in bilateral disease and lateral position with healthy lung down in case of unilateral infiltrates [19].

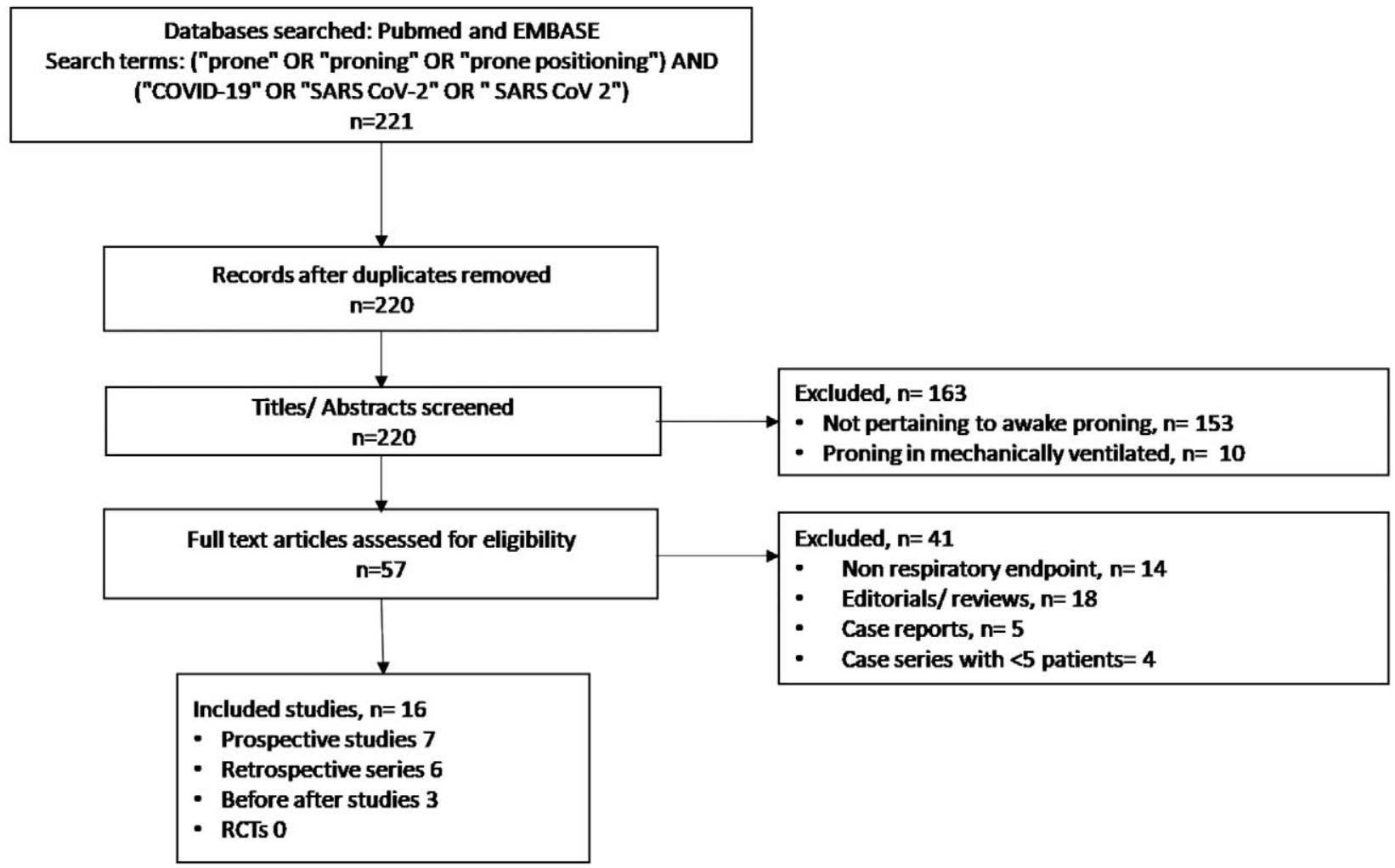

Figure 1. The flow-diagram for studies included in the systematic review and meta-analysis. 
Table 1. Characteristics of the studies included in the systematic review.

\begin{tabular}{|c|c|c|c|c|c|c|c|c|c|c|}
\hline No. & Authors & $\begin{array}{l}\text { Number } \\
\text { of } \\
\text { patients }\end{array}$ & Intervention & Setting & Outcomes & $\begin{array}{l}\text { Characteristics } \\
\text { of included } \\
\text { patients } \\
\text { Mean age } \\
\text { Female, n (\%) }\end{array}$ & Interface & $\begin{array}{l}\text { Prone } \\
\text { duration }\end{array}$ & Key results* & Remarks \\
\hline 1 & $\begin{array}{l}\text { Caputo et al. } \\
{[18]}\end{array}$ & 50 & Awake PP & $\begin{array}{l}\text { New York } \\
\text { Prospective } \\
\text { cohort } \\
\text { study } \\
\text { Patients } \\
\text { with } \\
\text { hypoxia on } \\
\text { arrival in } \\
\text { Emergency } \\
\text { Department }\end{array}$ & $\begin{array}{l}\mathrm{SpO}_{2} \text { before } \\
\text { and five } \\
\text { minutes after } \\
\mathrm{PP}\end{array}$ & $\begin{array}{l}59 \text { years } \\
20(40 \%)\end{array}$ & $\begin{array}{l}\text { NRBM } \\
38, \mathrm{NC} \\
12\end{array}$ & NA & $\begin{array}{l}\text { Median (IQR) } \\
\text { SpO2 improved } \\
\text { from } 84(75-90) \text { to } \\
94 \%(90-95) \\
\text { Intubation rate: } \\
24 \% \text { at } 24 \text { hours, } \\
18 / 50 \text { overall } \\
\text { Death: NA }\end{array}$ & $\begin{array}{l}\text { Large sample } \\
\text { size } \\
\text { No defined } \\
\text { standard of care } \\
\text { Excluded } \\
\text { patients on NIV }\end{array}$ \\
\hline 2 & $\begin{array}{l}\text { Coppo et al. } \\
{[17]}\end{array}$ & 56 & $\begin{array}{l}\text { Awake PP } \\
\text { for } \geq 3 \mathrm{~h}\end{array}$ & $\begin{array}{l}\text { Italy } \\
\text { Prospective } \\
\text { cohort } \\
\text { study } \\
56 \text { patients } \\
\text { on } \\
\text { supplement } \\
\text { al } \\
\text { oxygen/CP } \\
\text { AP }\end{array}$ & $\begin{array}{l}\text { Variation in } \\
\text { oxygenation } \\
\mathrm{PaO}_{2}: \mathrm{FiO}_{2} \\
\text { between } \\
\text { baseline and } \\
\text { after } 1 \text { hour of } \\
\text { resupination, } \\
\text { as an index of } \\
\text { pulmonary } \\
\text { recruitment }\end{array}$ & $\begin{array}{l}57.4 \text { years } \\
12(26.1 \%)\end{array}$ & $\begin{array}{l}\text { CPAP } \\
(4) \\
\text { Reserv } \\
\text { oir } \\
\text { mask } \\
(9) \\
\text { Venturi } \\
\text { mask } \\
(3)\end{array}$ & $\begin{array}{l}3.5 \\
\text { hours/da } \\
\text { y }\end{array}$ & $\begin{array}{l}\mathrm{PaO}_{2}: \mathrm{FiO}_{2} \text { ratio } \\
180.5 \mathrm{~mm} \mathrm{Hg} \text { in } \\
\text { supine } v s 285.5 \mathrm{~mm} \\
\mathrm{Hg} \text { in prone } \\
\text { position }(\mathrm{p}<0.0001) \\
\text { Improvement was } \\
\text { maintained in half } \\
\text { patients after } 1 \mathrm{~h} \\
\text { resupination } \\
\text { (responders) } \\
\text { Awake PP feasible } \\
(\geq 3 \mathrm{~h}) \text { in } 47 \\
(83 \cdot 9 \%) \\
\text { Intubation rate: } \\
13 / 46 \\
\text { Death: } 5 / 46\end{array}$ & $\begin{array}{l}\text { Largest cohort } \\
\text { study } \\
\text { Done in various } \\
\text { settings } \\
\text { (ED/ward) } \\
\text { Low duration of } \\
\text { PP (mean } 3.5 \mathrm{~h} \\
\text { per day) } \\
\text { No use of } \\
\text { HFNC } \\
\text { Majority were } \\
\text { on CPAP so } \\
\text { effect of PAP } \\
\text { may cause bias }\end{array}$ \\
\hline 3 & $\begin{array}{l}\text { Damarla et } \\
\text { al. [29] }\end{array}$ & 10 & $\begin{array}{l}\text { Two hours } \\
\text { alternating } \\
\text { prone and } \\
\text { supine }\end{array}$ & $\begin{array}{l}\text { USA } \\
\text { Retrospecti } \\
\text { ve cohort } \\
\text { study } \\
\text { Single } \\
\text { center } \\
\text { ICU, non- } \\
\text { intubated } \\
\text { hypoxic } \\
\text { patients }\end{array}$ & $\begin{array}{l}\text { Change in } \\
\mathrm{Spo}_{2} \text { and } \mathrm{RR} \\
\text { after one hour } \\
\text { of PP }\end{array}$ & $\begin{array}{l}56.6 \text { years } \\
4(40 \%)\end{array}$ & $\begin{array}{l}1 \mathrm{RA}, \\
\mathrm{HFNC} \\
4, \mathrm{NC} 5\end{array}$ & NA & $\begin{array}{l}\mathrm{SpO}_{2} \text { change } 94 \% \\
\text { [IQR, 91-95\%] to } \\
98 \% \text { [IQR,97-99\%] } \\
\text { Respiratory rate } \\
\text { change } 31 \text { [IQR, } 28 \\
\text { to } 39] \text { to } 22 \text { [IQR, } \\
18 \text { to } 25] \text { per } \\
\text { minute } \\
\text { Intubation rate: } \\
\text { 2/10 } \\
\text { Death: } 0 / 10\end{array}$ & $\begin{array}{l}\text { No control } \\
\text { group. Small } \\
\text { sample size }\end{array}$ \\
\hline 4 & $\begin{array}{l}\text { Sartini et al. } \\
{[10]}\end{array}$ & 15 & $\begin{array}{l}\text { One hour } \\
\text { awake PP. } \\
\text { Continued } \\
\text { if } \\
\text { improvem } \\
\text { ent in } \\
\text { oxygenati } \\
\text { on in } 1 \\
\text { hour }\end{array}$ & $\begin{array}{l}\text { Milan, Italy } \\
\text { Before- } \\
\text { after study } \\
\text { Outside } \\
\text { ICU } \\
\text { Inclusion } \\
\text { criteria: } \\
\text { Poor } \\
\text { response to } \\
\text { NIV in } \\
\text { supine at } \\
\text { FiO2 } 0.6 \text {, } \\
\text { CPAP } 10 \\
\text { cm H2O }\end{array}$ & $\begin{array}{l}\mathrm{Spo}_{2} \text {, derived } \\
\mathrm{PaO}_{2}: \mathrm{Fio}_{2} \text {, } \\
\text { respiratory } \\
\text { rate, and } \\
\text { patient's } \\
\text { comfort using } \\
\text { a numerical } \\
\text { rating scale } \\
\text { ( } 0 \text {, totally } \\
\text { uncomfortabl } \\
\text { e, to } 10, \text { fully } \\
\text { comfortable) } \\
\text { measured } \\
\text { before NIV, } \\
\text { during NIV in } \\
\text { pronation (60 } \\
\text { minutes after } \\
\text { start), and } 60 \\
\text { minutes after } \\
\text { NIV end }\end{array}$ & $\begin{array}{l}59 \text { years } \\
2(13.3)\end{array}$ & NIV & $\begin{array}{l}3 \text { hours/ } \\
\text { day }\end{array}$ & $\begin{array}{l}\text { Oxygenation values } \\
\text { not available. All } \\
\text { patients had } \\
\text { improvement in } \\
\mathrm{RR}, \mathrm{SpO}_{2} \text {, and } \\
\mathrm{PaO}_{2}: \mathrm{FiO}_{2} \\
11(73.3 \%) \text { had an } \\
\text { improvement in } \\
\text { comfort during } \\
\text { pronation. } \\
14 \text { day follow up- } 3 \\
\text { continued PP } \\
\text { Intubation rate: } \\
1 / 15 \\
\text { Death: } 1 / 15\end{array}$ & $\begin{array}{l}14 \text { day follow } \\
\text { up reported } \\
\text { Mean duration } \\
\text { of prone } \\
\text { position is } 3 \\
\text { hours. } \\
\text { Short duration } \\
\text { of NIV } \\
\text { Lack of control } \\
\text { group }\end{array}$ \\
\hline
\end{tabular}


Table 1. Continued from previous page.

\begin{tabular}{|c|c|c|c|c|c|c|c|c|c|c|}
\hline No. & Authors & $\begin{array}{c}\text { Number } \\
\text { of } \\
\text { patients }\end{array}$ & Intervention & Setting & Outcomes & $\begin{array}{l}\text { Characteristics } \\
\text { of included } \\
\text { patients } \\
\text { Mean age } \\
\text { Female, n (\%) }\end{array}$ & Interface & $\begin{array}{l}\text { Prone } \\
\text { duration }\end{array}$ & Key results* & Remarks \\
\hline 5 & $\begin{array}{l}\text { Elharrar et } \\
\text { al. [11] }\end{array}$ & 24 & $\begin{array}{l}\text { Awake PP } \\
\text { as } \\
\text { tolerated }\end{array}$ & $\begin{array}{l}\text { France } \\
\text { Prospective } \\
\text { cohort } \\
\text { study } \\
\text { Single } \\
\text { center } \\
\text { Requiring } \\
\text { oxygen and } \\
\text { CT } \\
\text { showing } \\
\text { posterior } \\
\text { lesions }\end{array}$ & $\begin{array}{l}\text { Proportion of } \\
\text { responders } \\
\text { i.e. } \mathrm{PaO}_{2} \\
\text { increase } \\
\geq 20 \% \\
\text { between } \\
\text { before and } \\
\text { during PP. } \\
\text { Tolerance of } \\
\text { prone } \\
\text { positioning as } \\
\text { on Visual } \\
\text { Analogue } \\
\text { Scale }\end{array}$ & $\begin{array}{l}66.1 \text { years } \\
8(33.3)\end{array}$ & $\begin{array}{l}\text { COT } \\
(<41 / \mathrm{mi} \\
\mathrm{n})-16, \\
\text { HFNC } \\
\text { or } \\
>41 / \mathrm{min} \\
-8\end{array}$ & $\begin{array}{l}63 \% \\
\text { more } \\
\text { than } 3 \\
\mathrm{~h} / \text { day }\end{array}$ & $\begin{array}{l}\text { No significant } \\
\text { difference between } \\
\mathrm{PaO}_{2} \text { before } \mathrm{PP} \text { and } \\
\mathrm{PaO}_{2} \text { after } \\
\text { resupination } \\
25 \% \text { responders } \\
63 \% \text { able to tolerate } \\
\mathrm{PP} \text { for } \geq 3 \mathrm{~h} \\
\text { Intubation rate: } \\
5 / 24 \\
\text { Death: NA }\end{array}$ & $\begin{array}{l}\text { Reported patient } \\
\text { tolerance to PP } \\
\text { Small study } \\
\text { Single episode } \\
\text { of PP was } \\
\text { evaluated }\end{array}$ \\
\hline 6 & $\begin{array}{l}\text { Moghadam } \\
\text { et al. }[23]\end{array}$ & 10 & Awake PP & $\begin{array}{l}\text { Iran } \\
\text { Before- } \\
\text { after study } \\
\text { COVID-19 } \\
\text { ward }\end{array}$ & $\begin{array}{l}\mathrm{SpO}_{2} \text { before } \\
\text { and after } \\
\text { prone } \\
\text { positioning }\end{array}$ & $\begin{array}{l}41 \text { years } \\
3(30 \%)\end{array}$ & NA & NA & $\begin{array}{l}\text { Mean (SD) } \mathrm{SpO}_{2} \\
\text { improved from } 85.6 \\
(0.69) \% \text { to } 95.9 \\
(2.2) \% \text {. } \\
\text { Feeling of dyspnea } \\
\text { decreased to } 40 \% \\
\text { Intubation: } 0 \\
\text { Death: } 0\end{array}$ & $\begin{array}{l}\text { Duration of PP } \\
\text { not available } \\
\text { No control } \\
\text { group }\end{array}$ \\
\hline 7 & $\begin{array}{l}\text { Golestaniera } \\
\text { ghi et al. } \\
{[21]}\end{array}$ & 10 & $\begin{array}{l}\text { Awake PP } \\
\text { in } 2 \mathrm{~h} \\
\text { cycles or } \\
\text { as per } \\
\text { tolerability }\end{array}$ & $\begin{array}{l}\begin{array}{l}\text { Iran } \\
\text { Before- } \\
\text { after study }\end{array} \\
\mathrm{PaO}_{2}: \mathrm{FiO}_{2} \\
<150 \text { on } \\
\text { helmet NIV }\end{array}$ & $\begin{array}{l}\text { Oxygenation } \\
\text { before and } \\
\text { after proning }\end{array}$ & NA & $\begin{array}{l}\text { Helmet } \\
\text { NIV }\end{array}$ & $\begin{array}{l}9 \text { hours/ } \\
\text { day }\end{array}$ & $\begin{array}{l}\text { Sustained } \\
\text { improvement ( }>12 \\
\mathrm{~mm} \mathrm{Hg} \text { ) in } \\
\text { oxygenation in } 60 \% \\
\text { cases } \\
\text { No adverse events } \\
\text { Intubation: } 2 / 10 \\
\text { Death: } 2 / 10\end{array}$ & $\begin{array}{l}\text { Higher mean } \\
\text { duration of PP } \\
\text { No control } \\
\text { group }\end{array}$ \\
\hline 8 & $\begin{array}{l}\mathrm{Ng} \text { et al. } \\
\text { [22] }\end{array}$ & 10 & $\begin{array}{l}\text { Awake PP } \\
1 \text { hour } \\
\text { each } \\
\text { session, } \\
\text { five } \\
\text { sessions a } \\
\text { day, three } \\
\text { hours } \\
\text { apart }\end{array}$ & $\begin{array}{l}\text { Singapore } \\
\text { Before } \\
\text { after study } \\
\text { General } \\
\text { ward } \\
\text { COVID-19 } \\
\text { related } \\
\text { hypoxia } \\
\text { with } \mathrm{FiO}_{2}< \\
50 \% \\
\end{array}$ & $\begin{array}{l}\text { Descriptive } \\
\text { study }\end{array}$ & $\begin{array}{l}60.6 \text { years } \\
2(20 \%)\end{array}$ & $\mathrm{NC}$ & $\begin{array}{l}\text { Cumulati } \\
\text { ve } \\
\text { median } \\
21 \text { h over } \\
\text { median } 8 \\
\text { days }\end{array}$ & $\begin{array}{l}\text { Oxygenation } \\
\text { improvement: } 70 \% \\
\text { Intubation: } 1 / 10 \\
\text { Death: } 1 / 10\end{array}$ & $\begin{array}{l}\text { Protocolized } \\
\text { proning done } \\
\text { Detailed } \\
\text { oxygenation } \\
\text { parameters not } \\
\text { reported }\end{array}$ \\
\hline 9 & $\begin{array}{l}\text { Xu et al. } \\
\text { [28] }\end{array}$ & 10 & $\begin{array}{l}\text { Awake PP } \\
\text { with target } \\
\text { prone } 16 \\
\text { h/day }\end{array}$ & $\begin{array}{l}\text { China } \\
\text { Retrospecti } \\
\text { ve cohort } \\
\text { study } \\
\text { Early } \\
\text { awake PP } \\
\text { combined } \\
\text { with HFNC }\end{array}$ & $\begin{array}{l}\text { Oxygenation } \\
\text { improvement } \\
\text { Survival }\end{array}$ & $\begin{array}{l}50.2 \\
5(50 \%)\end{array}$ & HFNC & NA & $\begin{array}{l}\text { Significant } \\
\text { improvement in } \\
\text { median } \mathrm{PaO}_{2}: \mathrm{FiO}_{2} \\
\text { (exact numbers not } \\
\text { available) } \\
\text { Median (IQR) } \\
\mathrm{PaCO}_{2} \text { increased } \\
\text { slightly [32.3(29.3- } \\
\text { 34.0) vs } 29.7(28.0- \\
\text { 32.0), p<0.001] } \\
\text { Intubation:0 } \\
\text { Death: } 0\end{array}$ & $\begin{array}{l}\text { Larger target } \\
\text { mean duration } \\
\text { of PP } \\
\text { Exact PP } \\
\text { duration not } \\
\text { reported }\end{array}$ \\
\hline
\end{tabular}


Table 1. Continued from previous page.

\begin{tabular}{|c|c|c|c|c|c|c|c|c|c|c|}
\hline No. & Authors & $\begin{array}{c}\text { Number } \\
\text { of } \\
\text { patients }\end{array}$ & Intervention & Setting & Outcomes & $\begin{array}{l}\text { Characteristics } \\
\text { of included } \\
\text { patients } \\
\text { Mean age } \\
\text { Female, n (\%) }\end{array}$ & Interface & $\begin{array}{l}\text { Prone } \\
\text { duration }\end{array}$ & Key results* & Remarks \\
\hline 10 & $\begin{array}{l}\text { Thompson et } \\
\text { al. [27] }\end{array}$ & 25 & $\begin{array}{l}\text { Awake PP } \\
\text { as long as } \\
\text { tolerated } \\
\text { up to } 24 \\
\text { hours a } \\
\text { day }\end{array}$ & $\begin{array}{l}\text { USA } \\
\text { Retrospecti } \\
\text { ve cohort } \\
\text { study } \\
\text { Severe } \\
\text { hypoxemia } \\
\text { (respiratory } \\
\text { rate of } 30 \\
\text { breaths/min } \\
\text { or greater } \\
\text { and Spo of } \\
93 \% \text { or less } \\
\text { while } \\
\text { receiving } \\
\text { supplement } \\
\text { al oxygen } 6 \\
\text { L/min via } \\
\text { nasal } \\
\text { cannula } \\
\text { and } 15 \\
\text { L/min via } \\
\text { non- } \\
\text { rebreather } \\
\text { face mask) }\end{array}$ & $\begin{array}{l}\text { Change in } \\
\mathrm{Spo}_{2} \text { before } \\
\text { and } 1 \text { hour } \\
\text { after initiation } \\
\text { of the prone } \\
\text { position }\end{array}$ & $\begin{array}{l}66 \text { years } \\
7(28 \%)\end{array}$ & $\begin{array}{l}\mathrm{NC} \text { and } \\
\mathrm{NRBM}\end{array}$ & $\begin{array}{l}5 \\
\text { hours/da } \\
y\end{array}$ & $\begin{array}{l}\text { Median (SE) } \\
\text { increase in } \mathrm{SpO}_{2} \\
7 \%(1.2 \%) \\
\text { Range of } \\
\text { improvement in } \\
\mathrm{Spo}_{2} 1 \% \text { to } 34 \% \\
\text { Intubation rate: } 12 / \\
25 \\
\text { Death: } 3 / 25\end{array}$ & $\begin{array}{l}\text { Reported } \\
\text { intubation rate } \\
\text { difference as per } \\
\text { demographic } \\
\text { characteristics } \\
\text { Lack of control } \\
\text { group } \\
\text { Small Sample } \\
\text { size }\end{array}$ \\
\hline 11 & $\begin{array}{l}\text { Despres et } \\
a l .[26]\end{array}$ & 6 & $\begin{array}{l}\text { Awake PP } \\
\text { duration 1- } \\
16 \text { hours }\end{array}$ & $\begin{array}{l}\text { France } \\
\text { Retrospecti } \\
\text { ve case } \\
\text { series } \\
\text { HFNO or } \\
\text { convention } \\
\text { al oxygen } \\
\text { therapy } \\
\mathrm{SpO}_{2} \leq 92 \% \\
\text { at oxygen } \\
\geq 51 / \mathrm{min} \\
\end{array}$ & $\begin{array}{l}\text { Change in } \mathrm{P} / \mathrm{F} \\
\text { ratio }\end{array}$ & $\begin{array}{l}60 \text { years } \\
\text { All male }\end{array}$ & $\begin{array}{l}\text { HFNC } \\
3, \text { COT } \\
3\end{array}$ & $\begin{array}{l}3 \\
\text { hours/da } \\
y\end{array}$ & $\begin{array}{l}\mathrm{PaO}_{2}: \mathrm{FiO}_{2} \text { ratio } \\
\text { improved in } 4 \text { out } \\
\text { of } 6 \text { cases after } \mathrm{PP} \\
\text { Intubation rate: } 3 / 6 \\
\text { Death: Not } \\
\text { available }\end{array}$ & $\begin{array}{l}\text { No control } \\
\text { group } \\
\text { Small sample } \\
\text { size }\end{array}$ \\
\hline 12 & $\begin{array}{l}\text { Tu et al. } \\
{[20]}\end{array}$ & 9 & Awake PP & $\begin{array}{l}\text { China } \\
\text { Prospective } \\
\text { cohort } \\
\text { study } \\
\mathrm{HFNC \text {for }} \\
>2 \text { days, } \\
\text { and } \mathrm{PaO}_{2} \text { : } \\
\mathrm{FiO}_{2}<150\end{array}$ & $\begin{array}{l}\mathrm{PaO}_{2}, \mathrm{SpO}_{2} \\
\text { and } \mathrm{PaCO}_{2} \\
\text { before and } \\
\text { after PP }\end{array}$ & $\begin{array}{l}51 \text { years } \\
5(55.6 \%)\end{array}$ & HFNC & $\begin{array}{l}2 \\
\text { hours/da } \\
y\end{array}$ & $\begin{array}{l}\text { Mean (SD) } \\
\text { improvement in } \mathrm{SpO}_{2} \text { from } 90(2) \text { to } \\
96(3) \%(\mathrm{p}<0.001) \\
\text { Mean } \\
(\mathrm{SD}) \text { improvement } \\
\text { in } \mathrm{PaO}_{2} \text { from } 9(10) \\
\text { to } 108(14) \mathrm{mmHg} \\
(\mathrm{P}<0.001) \\
\text { Decrease in mean } \\
(\mathrm{SD}) \mathrm{PaCO}_{2} \text { from } \\
47(7) \text { to } 39(5) \\
\text { mmHg }(\mathrm{P}=0.007) \\
\text { Intubation: } 2 / 9 \\
\text { Death: Not } \\
\text { available }\end{array}$ & $\begin{array}{l}\text { Low median } \\
\text { duration of } \\
\text { awake PP, } \\
\text { awake PP used } \\
\text { as salvage } \\
\text { therapy }\end{array}$ \\
\hline
\end{tabular}

To be continued on next page 
Table 1. Continued from previous page.

\begin{tabular}{|c|c|c|c|c|c|c|c|c|c|c|}
\hline No. & Authors & $\begin{array}{c}\text { Number } \\
\text { of } \\
\text { patients }\end{array}$ & Intervention & Setting & Outcomes & $\begin{array}{l}\text { Characteristics } \\
\text { of included } \\
\text { patients } \\
\text { Mean age } \\
\text { Female, n (\%) }\end{array}$ & Interface & $\begin{array}{l}\text { Prone } \\
\text { duration }\end{array}$ & Key results* & Remarks \\
\hline 13 & $\begin{array}{l}\text { Retucci et } \\
\text { al. [19] }\end{array}$ & 26 & $\begin{array}{l}\text { Awake PP } \\
\text { in bilateral } \\
\text { disease } \\
\text { Lateral } \\
\text { positionin } \\
\mathrm{g} \text { in } \\
\text { unilateral } \\
\text { disease }\end{array}$ & $\begin{array}{l}\text { Italy } \\
\text { Prospective } \\
\text { cohort } \\
\text { study } \\
\mathrm{PaO}_{2}: \mathrm{FIO}_{2} \\
\text { during } \\
\text { helmet } \\
\mathrm{CPAP} \\
<250 \text { after } \\
\text { at least } 48 \\
\text { hours }\end{array}$ & $\begin{array}{l}\text { Primary } \\
\text { outcome was } \\
\text { the success of } \\
\text { the } \\
\text { prone/lateral } \\
\text { positioning } \\
\text { trial, defined } \\
\text { as the } \\
\text { occurrence of } \\
\text { all of the } \\
\text { following } \\
\text { criteria at T1 } \\
\text { in comparison } \\
\text { with T0: (1) a } \\
\text { decrease of } \\
\text { A-aO }{ }_{2} \text { of at } \\
\text { least } 20 \%,(2) \\
\text { equal or } \\
\text { reduced } \\
\text { respiratory } \\
\text { rate, }(3) \text { equal } \\
\text { or reduced } \\
\text { Dyspnea } \\
\text { BORG scale), } \\
\text { (4) } \mathrm{SBP}>90 \\
\text { mm } \mathrm{Hg} \text {. }\end{array}$ & \begin{tabular}{|l|}
62 years \\
$9(34.6 \%)$
\end{tabular} & $\begin{array}{l}\text { Helmet } \\
\text { CPAP }\end{array}$ & NA & $\begin{array}{l}15.4 \% \text { successful } \\
\text { with a decrease of } \\
\mathrm{A}-\mathrm{aO}_{2} \text { of } 20 \% \text { or } \\
\text { more during the } \\
\text { trial in comparison } \\
\text { with baseline. } \\
\text { Seventeen trials } \\
(46.1 \%) \text { showed a } \\
\text { decrease of }<20 \% \\
\text { ofA-aO } 2 \\
25 \% \text { (awake PP) } \\
\text { and } 40 \% \text { (lateral } \\
\text { positioning failed } \\
\text { Intubation: } 7 / 26 \\
\text { Death: } 2 / 26\end{array}$ & $\begin{array}{l}\text { Lateral } \\
\text { positioning } \\
\text { employed } \\
\text { Excluded } \mathrm{SpO}_{2} \\
<90 \% \text { at } \mathrm{FIO}_{2} \\
>0.8\end{array}$ \\
\hline 14 & $\begin{array}{l}\text { Zang et al. } \\
{[6]}\end{array}$ & 60 & $\begin{array}{l}23 \text { Awake } \\
\text { PP } \\
37 \\
\text { controls }\end{array}$ & $\begin{array}{l}\text { China } \\
\text { Prospective } \\
\text { cohort } \\
\text { study } \\
86 \% \leq \\
\mathrm{SpO}_{2} \leq 93 \% \\
\text { on } \mathrm{NRBM} \\
101 / \mathrm{min}\end{array}$ & $\begin{array}{l}\mathrm{SpO}_{2}, \mathrm{RR}, \\
\mathrm{ROX} \text { index at } \\
10 \text { min and } 30 \\
\text { min of PP } \\
90 \text { d mortality }\end{array}$ & $\begin{array}{l}62.7 \text { years } \\
10(16.7 \%)\end{array}$ & NRBM & $\begin{array}{l}9 \text { hours } \\
\text { cumulati } \\
\text { ve }\end{array}$ & $\begin{array}{l}\text { Significant } \\
\text { improvement in } \\
\mathrm{SpO}_{2}, \mathrm{RR}, \mathrm{ROX} \\
\text { index at } 10 \text { min and } \\
30 \text { min of awake } \\
\mathrm{PP} . \\
90 \text { days of follow- } \\
\text { up, } 10 \text { (43.5\%) died } \\
\text { in the awake PP } \\
\text { group, } 28(75.7 \%) \\
\text { in non-prone } \\
\text { position group } \\
\text { Intubation rate: } 8 / \\
23 \\
\text { Death: } 10 / 23\end{array}$ & $\begin{array}{l}\text { 90d mortality } \\
\text { assessed } \\
\text { Presence of } \\
\text { control group } \\
\text { Non randomized }\end{array}$ \\
\hline 15 & $\begin{array}{l}\text { Ripoll- } \\
\text { Gallardo et } \\
\text { al. }[25]\end{array}$ & 13 & Awake PP & $\begin{array}{l}\text { Italy } \\
\text { Retrospecti } \\
\text { ve cohort } \\
\text { study } \\
\text { CPAP with } \\
0.6 \mathrm{FiO}_{2} \\
\text { and } 10 \\
\mathrm{CMH} 20 \\
\mathrm{PEEP} \text { and } \\
\text { pronated if } \\
\mathrm{PaO}_{2}: \mathrm{FiO}_{2} \\
<150 \\
\mathrm{mmHg}^{-}\end{array}$ & $\begin{array}{l}12 / 13 \\
\text { improved } \\
\text { oxygenation }\end{array}$ & $\begin{array}{l}66.3 \text { years } \\
2(15.3 \%)\end{array}$ & CPAP & $\begin{array}{l}2.4 \\
\text { hours/ } \\
\text { day }\end{array}$ & $\begin{array}{l}\mathrm{PaO}_{2}: \mathrm{FiO}_{2} \\
\text { improved from } \\
115.2 \pm 13.3 \text { to } \\
166.4 \pm 70 \\
\text { Intubation rate: } 9 / \\
13 \\
\text { Death: } 7 / 13\end{array}$ & Sicker cohort \\
\hline 16 & $\begin{array}{l}\text { Huang et al. } \\
{[24]}\end{array}$ & 29 & Awake PP & $\begin{array}{l}\text { China } \\
\text { Retrospecti } \\
\text { ve cohort } \\
\text { study } \\
\text { Series of } \\
60 \text { patients } \\
\text { of severe } \\
\text { COVID-19 }\end{array}$ & No mortality & NA & NA & NA & $\begin{array}{l}\mathrm{PaO}_{2}: \mathrm{FiO}_{2} \\
\text { improved from } \\
158.7 \text { to } 237.3 \\
\text { Intubation rate: not } \\
\text { available } \\
\text { Death: None }\end{array}$ & $\begin{array}{l}\text { Awake PP data } \\
\text { not separately } \\
\text { provided }\end{array}$ \\
\hline
\end{tabular}




\section{Quality assessment}

Eight studies were classified as limited quality, while four strong, one good, and three were adjudged of adequate quality. The mean (SD) score on Qualsys was 0.58 (0.22) (Supplementary Table 1). Only one study had included a control group; however, the method of group allocation was not clear [6]. The study flow diagram is shown in Figure 1.

\section{Intubation rate}

Among the 316 patients included in the review, the incidence of intubation was reported in all except one study. Out of the 287 patients for whom intubation outcome was available, 83 patients $(28.9 \%)$ required IMV. The overall pooled proportion of patients who required IMV was 0.25 (95\% confidence interval $0.16-0.34)$ (Figure 2). There was significant heterogeneity among studies reporting intubation rate $\left(\mathrm{I}^{2}=62.5 \%\right)$ and there was no publication bias as assessed by funnel plot (Supplementary Figure 1).

\section{Oxygenation indices}

Most of the studies report a significant improvement in oxygenation status as measured by $\mathrm{PaO} 2$ : FiO2 ratio, $\mathrm{PaO} 2$, pulse oxygen saturation, and respiratory rate. The percentage of patients who exhibit improvement in oxygenation status after awake PP varied widely from $25 \%$ to $100 \%$, as varying criteria were used for defining improvement Prone positioning yielded a significant medium effect size for overall oxygenation improvement measured by any of the parameters (SMD 1.72, 95\% CI 1.01-2.43) as depicted in Figure 3. In the two studies which reported oxygenation parameters after re-supination, the improvement in oxygenation was not sustained $[17,19]$.

\section{$\mathrm{PaO}_{2}: \mathrm{FiO}_{2}$}

The $\mathrm{PaO}_{2}: \mathrm{FiO}_{2}$ ratio was compared before and during PP in five studies [17,19,24-26]. Cumulatively, the weighted mean difference in $\mathrm{PaO}_{2}: \mathrm{FiO}_{2}$ ratio after and before prone positioning was $51.29 \mathrm{mmHg}(95 \% \mathrm{CI} 13.91-88.67)$ in four studies with complete data (Figure 4a). There was significant heterogeneity among the studies reporting a change in $\mathrm{PaO}_{2}: \mathrm{FiO}_{2}$ ratio (I2 $=72.4 \%)$.

\section{Partial pressure of oxygen in arterial blood $\left(\mathrm{PaO}_{2}\right)$}

The partial pressure of oxygen in arterial blood $\left(\mathrm{PaO}_{2}\right)$ before and during awake PP was reported in five studies $[10,11,18,20,23]$. The cumulative mean improvement (WMD) in $\mathrm{PaO}_{2}$ during prone positioning was $27.94 \mathrm{~mm} \mathrm{Hg}$ (95\% CI 15.2040.69) (Figure 4b).

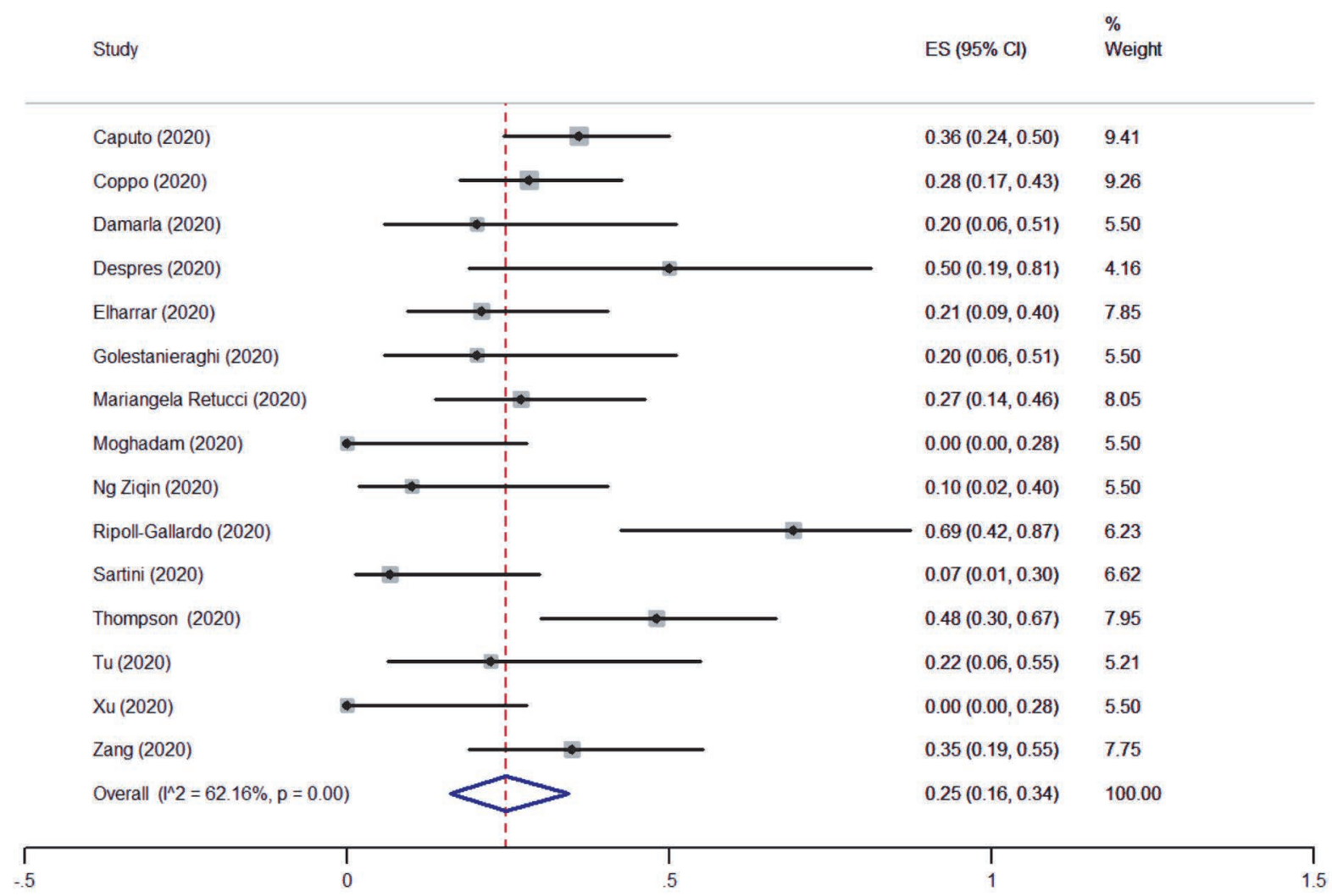

Figure 2. The forest plot depicting pooled proportion of patients requiring intubation and mechanical ventilation. 


\section{Pulse oxygen saturation $\left(\mathrm{SpO}_{2}\right)$}

Pulse oxygen saturation $\left(\mathrm{SpO}_{2}\right)$ before and during awake PP was described in seven studies $[6,17,18,20,23,27,29]$. The pooled effect, i.e. the mean difference in $\mathrm{SpO}_{2}$ after awake PP, from four studies providing mean and SD, was $5.39 \%$ (95\% CI 1.53-9.25) (Figure 4c) [6,17,20,23].

\section{Respiratory rate}

Respiratory rate change with awake PP was reported in six studies, and complete data were available for five of them. The mean change in RR following awake PP was -0.83 (95\% CI -3.02 to 1.37 ) (Figure 4d).

\section{ROX index}

$\mathrm{ROX}$ index $\left(\mathrm{SpO}_{2} / \mathrm{FiO}_{2}(\%) /\right.$ Respiratory rate) was reported in one study [6]. ROX index increased from $3.35 \pm 0.46$ to $3.96 \pm 0.45$ after $30 \mathrm{~min}$ of prone positioning $(\mathrm{p}<0.01)$.

\section{Mortality and length of stay}

Only one study with a control group reported the 90-day mortality [6]. A total of $10(43.5 \%)$ patients died in the awake PP group, compared with $28(75.7 \%)$ patients in the non-prone position group [6]. Twelve studies reported the number of deaths in the observational cohort, yielding a cumulative 31 deaths out of 227 patients $(13.7 \%)$. Length of stay was reported in four studies, yielding a median (IQR) of 12.9 (5.6- 25.4) days.

\section{Timing of awake PP initiation}

The time from hospitalization to awake PP was significantly different in responders and non-responders in one study $(2.7 \mathrm{~d} v \mathrm{~s}$ $4.6 \mathrm{~d})$ suggesting the role of employing awake PP early in the disease course [17].

\section{Safety}

Most safety data were of low quality from single-group studies. In the study by Elharrar et al., $63 \%$ of patients were able to tolerate awake PP for $\geq 3$ hours, while another study showed feasibility in up to $83.9 \%[11,17]$. Back pain was reported in $42 \%$ during awake PP [11]. Episodes of hypotension or desaturation were not observed in any of the studies. One series reported half the patients showing a decrease in $\mathrm{PaO}_{2}$ : $\mathrm{FiO}_{2}$ ratio when awake PP was used in severe COVID-19 patients [26]. One study analyzed comfort and dyspnea before and during awake PP by visual analog scale (VAS) and reported an improvement from 3 to 2 in dyspnea and an increase in discomfort from 0 to 4 median [11]. Failure of awake $\mathrm{PP}$ in terms of increased respiratory rate or elevation of the alveolar-arterial gradient happened in $25 \%$ in prone positioning to $40 \%$ in the lateral position [19]. No studies reported any pressure sores.

\section{Discussion}

Evidence from the available studies indicates that awake PP used along with non-invasive oxygen delivery devices improves oxygenation in patients with COVID-19 related acute hypoxemic respiratory failure. The intubation rate in this pooled cohort was $28.9 \%$. As per a recent review, among COVID-19 patients admitted to ICU, 35.4-100\% require invasive mechanical ventilation [30]. This difference in intubation rate can not be directly attributed to awake PP, but it does suggest a lack of harm as there was no increase in the intubation rate in the patients undergoing awake PP. When added to high-flow nasal cannula (HFNC) or NIV, early prone positioning has been shown to avoid the need for intubation in almost half of the patients with moderate to severe ARDS, even in non-COVID-19 patients [8]. Improvement in oxygenation alone may not lead to improved clinical outcomes as demonstrated in previous trials of inhaled nitric oxide, surfactant therapy, and higher tidal volumes which improved oxygenation but did not produce

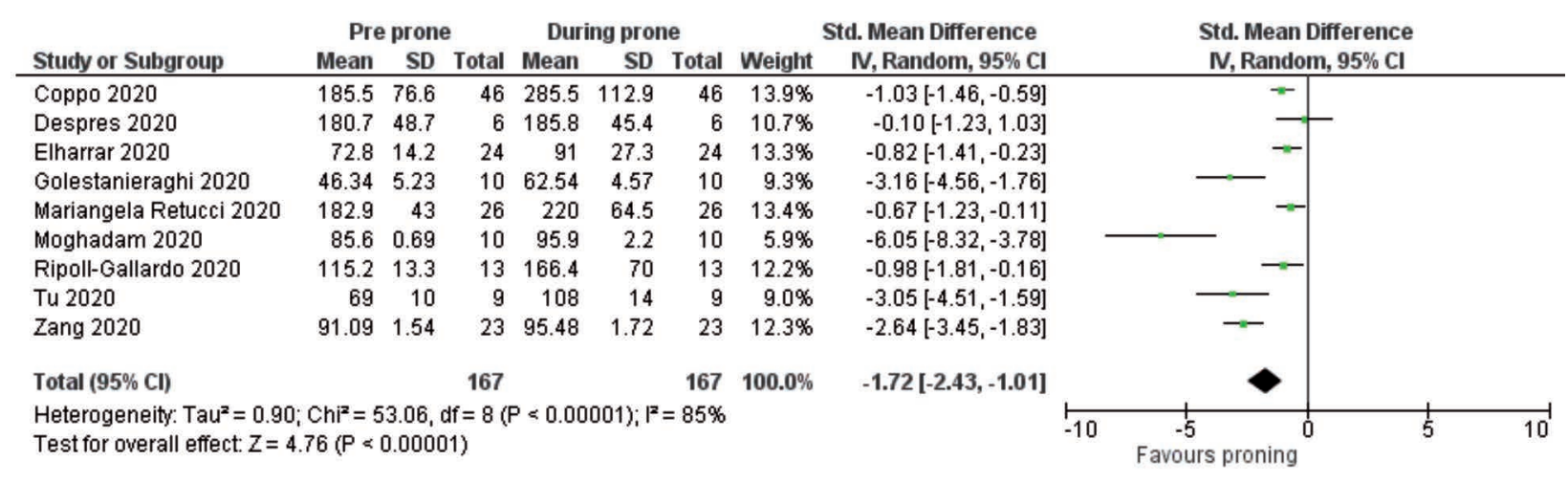

Figure 3. The first plot depicting pooled difference in any of the oxygenation parameter as measured by standardized mean difference during awake PP. Four studies (Coppo, Despres, Mariangela Retucci and Ripoll-Gallardo) reported $\mathrm{PaO}_{2}: \mathrm{FiO}_{2}$, three studies (Elharrar, Golestanieraghi and $\mathrm{Tu}$ ) reported $\mathrm{PaO}_{2}$ and two studies (Moghandam and $\mathrm{Zang}$ ) reported $\mathrm{SpO}_{2}$. 
any clinically relevant outcomes in larger trials [31]. A critical finding from this meta-analysis is improved oxygenation by awake PP; however, no conclusion regarding other clinically relevant outcomes such as the need for IMV or length of stay could be withdrawn. The effect of awake PP on these outcomes needs further studies with a comparison group, ideally in a randomized fashion.

There remains a concern whether awake PP may delay intubation and cause harm which can not be concluded from the available evidence. There was a wide variation in the severity of hypoxemia in the included studies, leading to a variable rate of intubation.

No conclusion can be drawn about the minimum duration of awake PP required for clinical improvement as no study compared varying durations of awake PP and included studies had heterogeneity in the durations employed. We initially planned analysis to assess the effect of the duration of awake PP on oxygenation and avoidance of intubation; however, this was not possible due to lack of required data of duration of awake PP. The subset of patients who will benefit from awake PP is also hard to conclude, but there is an indication that early awake PP may have better results [17]. All studies have excluded patients requiring emergent intubation. The effect of concomitant drug therapy on the clinical outcomes studied could not be estimated because of the data's non-availabil- ity. We could not assess the effect of the application of CPAP over the conventional mode of oxygenation. As CPAP might correct hypoxemia more than standard oxygen delivery, it remains known whether patients receiving CPAP therapy had greater oxygenation improvement $[32,33]$. The study by Sartini et al. [10] described the combined effect of NIV and prone positioning; thus, it was not possible to separate the effect of NIV from the awake PP.

The pre-requisite for awake PP is an alert and conscious patient who can cooperate with position changes. Contraindications for awake PP include the requirement of urgent intubation, agitation or altered mental status, unstable spine or thoracic injury, recent abdominal surgery, and morbid obesity [34]. Hemodynamic instability is an absolute contraindication as a cardiac arrest without a definitive airway in the prone position may prove difficult for resuscitation as the patient will have to be supinated for securing the airway. Second or third-trimester pregnancy is a relative contraindication for awake PP, though case reports of the same have been published [35]. Tolerance is a concern with some studies reporting an inability to tolerate awake PP in a significant proportion. Awake PP requires close monitoring from the nursing staff to ensure it is successful and tolerated, especially during the initial sessions.

The major limitation of this systematic review is that we also

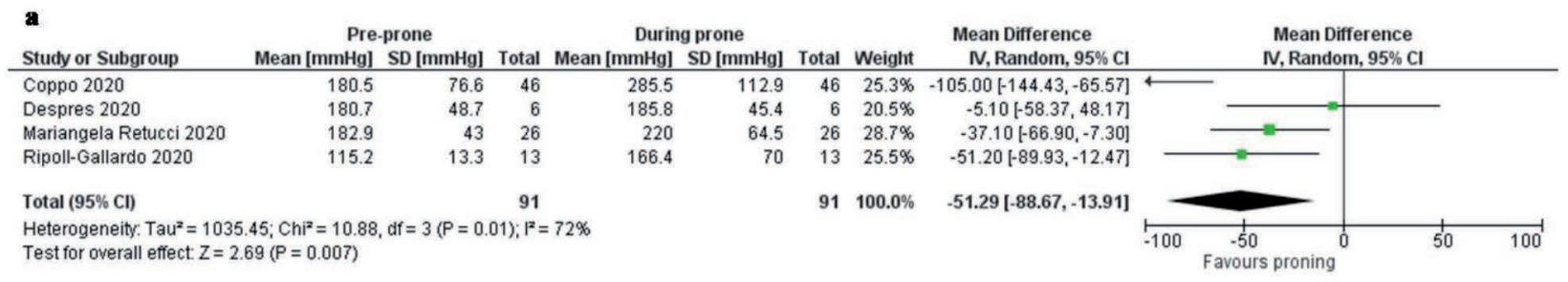

b

\begin{tabular}{|c|c|c|c|c|c|c|c|c|c|c|c|c|}
\hline \multirow[b]{2}{*}{ Study or Subgroup } & \multicolumn{3}{|c|}{ Pre-prone } & \multicolumn{3}{|c|}{ During Prone } & \multicolumn{3}{|c|}{ Mean Difference } & \multirow{2}{*}{\multicolumn{2}{|c|}{$\begin{array}{c}\text { Mean Difference } \\
\text { IV, Random, } 95 \% \mathrm{Cl}\end{array}$}} & \\
\hline & Mean & SD & Total & Mean & SD & Total & Weight & IV, Random, $95 \% \mathrm{Cl}$ & & & & \\
\hline Coppo 2020 & 117.1 & 47.4 & 46 & 200.4 & 110.9 & 46 & $8.9 \%$ & $-83.30[-118.15,-48.45]$ & $\longleftarrow$ & 工 & & \\
\hline Elharrar 2020 & 72.8 & 14.2 & 24 & 91 & 27.3 & 24 & $21.3 \%$ & $-18.20[-30.51,-5.89]$ & & $\rightarrow-$ & & \\
\hline Golestanieraghi 2020 & 46.34 & 5.23 & 10 & 62.54 & 4.57 & 10 & $25.8 \%$ & $-16.20[-20.50,-11.90]$ & & $=$ & & \\
\hline Mariangela Retucci 2020 & 86.9 & 15.1 & 26 & 104.6 & 25 & 26 & $22.0 \%$ & $-17.70[-28.93,-6.47]$ & & $\rightarrow-$ & & \\
\hline Tu 2020 & 69 & 10 & 9 & 108 & 14 & 9 & $22.0 \%$ & $-39.00[-50.24,-27.76]$ & & -- & & \\
\hline Total $(95 \% \mathrm{Cl})$ & & & 115 & & & 115 & $100.0 \%$ & $-27.94[-40.69,-15.20]$ & & & & \\
\hline $\begin{array}{l}\text { Heterogeneity: } \operatorname{Tau}^{2}=159 \\
\text { Test for overall effect: } Z=\end{array}$ & $\begin{array}{l}1 ; C^{2} i^{2} \\
30(P<\end{array}$ & $\begin{array}{l}=26.8 \\
0.0001\end{array}$ & $d f=$ & $4(P<0$. & 0001); $\mathrm{l}^{2}$ & $=85 x$ & & & -100 & $\begin{array}{l}-50 \\
\text { avours proning }\end{array}$ & 50 & 100 \\
\hline
\end{tabular}

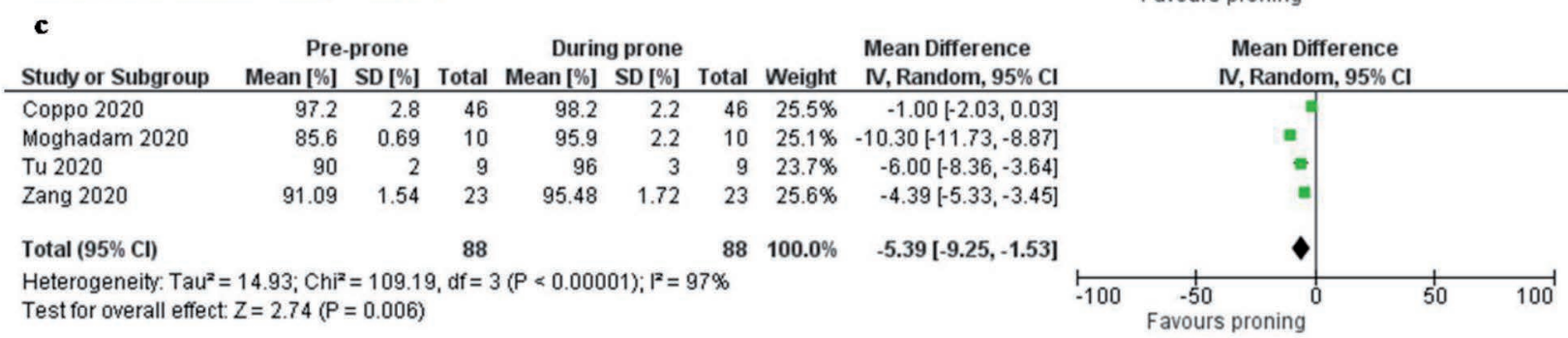

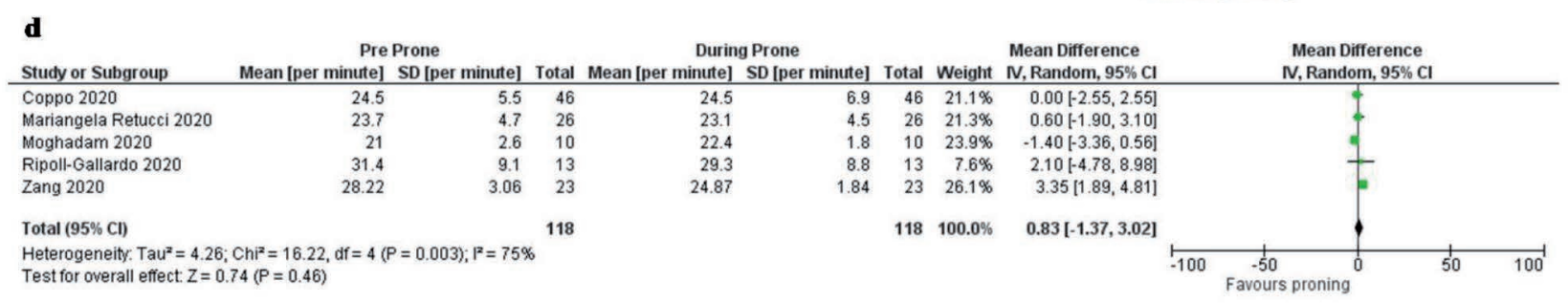

Figure 4. Forest plots depicting change in various parameters as weighted mean difference during awake $\mathrm{PP}$ a) $\mathrm{PaO}_{2}: \mathrm{FiO}_{2}$ ratio. b) Partial pressure of oxygen $\left(\mathrm{PaO}_{2}\right)$. c) Pulse oxygen saturation $\left(\mathrm{SpO}_{2}\right)$. d) Respiratory rate. 
included studies with lower quality. As the evidence for awake PP is still sparse and emerging, we attempted to summarize the evidence rather than derive definite conclusions. As there was only one study with a control group, it was not possible to derive a conclusion regarding awake PP compared to patients who did not undergo awake PP. In the current scenario of the ongoing pandemic, further studies regarding awake PP will likely continue to be conducted, and RCTs are being conducted as well (NCT04395144). Another major limitation is the substantial heterogeneity found in the meta-analysis. It is likely due to variable inclusion criteria and the duration of proning in the studies included in this meta-analysis. Due to this heterogeneity, we need to use caution in interpreting these results. However, as all studies demonstrated improved oxygenation, it is likely real benefit though the degree of improvement may vary.

\section{Conclusions}

Awake PP in non-intubated patients with COVID-19 hypoxemic respiratory failure might be associated with a reduction in the need for intubation and improvement in oxygenation. However, its effect on reducing mortality is still unclear. Awake PP is one strategy that has been widely advertised as low risk and inexpensive. Though available evidence is supportive, more studies, especially randomized trials, are required before this can become a routine procedure in hypoxic respiratory failure.

\section{References}

1. Richardson S, Hirsch JS, Narasimhan M, et al. Presenting characteristics, comorbidities, and outcomes among 5700 patients hospitalized with COVID-19 in the New York City area. JAMA 2020;323:2052-9.

2. Phua J, Weng L, Ling L, et al. Intensive care management of coronavirus disease 2019 (COVID-19): challenges and recommendations. Lancet Respir Med 2020;8:506-17.

3. Tzotzos SJ, Fischer B, Fischer H, Zeitlinger M. Incidence of ARDS and outcomes in hospitalized patients with COVID-19: a global literature survey. Crit Care 2020;24:1-4.

4. Munshi L, Del Sorbo L, Adhikari NKJ, et al. Prone position for acute respiratory distress syndrome. A systematic review and meta-analysis. Ann Am Thorac Soc 2017;14:S280-8.

5. Guérin C. Prone ventilation in acute respiratory distress syndrome. Eur Respir Rev 2014;23:249-57.

6. Zang X, Wang Q, Zhou H, et al. Efficacy of early prone position for COVID-19 patients with severe hypoxia: a single-center prospective cohort study. Intensive Care Med 2020;46:1927-9.

7. Sun Q, Qiu H, Huang M, Yang Y. Lower mortality of COVID19 by early recognition and intervention: experience from Jiangsu Province. Ann Intensive Care 2020;10:33.

8. Ding L, Wang L, Ma W, He H. Efficacy and safety of early prone positioning combined with HFNC or NIV in moderate to severe ARDS: a multi-center prospective cohort study. Crit Care 2020;24:28.

9. Scaravilli V, Grasselli G, Castagna L, et al. Prone positioning improves oxygenation in spontaneously breathing nonintubated patients with hypoxemic acute respiratory failure: A retrospective study. J Crit Care 2015;30:1390-4.

10. Sartini C, Tresoldi M, Scarpellini P, et al. Respiratory parame- ters in patients with COVID-19 after using noninvasive ventilation in the prone position outside the intensive care unit JAMA 2020;323:2338-40.

11. Elharrar X, Trigui Y, Dols A-M, et al. Use of prone positioning in nonintubated patients with COVID-19 and hypoxemic acute respiratory failure. JAMA 2020;323:2336-8.

12. Liberati A, Altman DG, Tetzlaff J, et al. The PRISMA statement for reporting systematic reviews and meta-analyses of studies that evaluate healthcare interventions: explanation and elaboration. BMJ 2009;339:b2700.

13. Chan K, Bhandari M. Three-minute critical appraisal of a case series article. Indian J Orthop 2011;45:103-4.

14. Lee L, Packer TL, Tang SH, Girdler S. Self-management education programs for age-related macular degeneration: a systematic review. Australas J Ageing 2008;27:170-6.

15. Kmet L M, Lee R C, Cook L S. Standard quality assessment criteria for evaluating primary research papers from a variety of fields. Edmonton: Alberta Heritage Foundation for Medical Research; 2004. Available from: https://www.crd.york.ac.uk/ CRDWeb/ShowRecord.asp?ID=32004000313\&ID=320040 00313.

16. The Cochrane Collaboration [Internet]. Higgins JPT, Thomas J, Chandler J, et al., editors. Cochrane Handbook for Systematic Reviews of Interventions. London: The Cochrane Collaboration.

17. Coppo A, Bellani G, Winterton D, et al. Feasibility and physiological effects of prone positioning in non-intubated patients with acute respiratory failure due to COVID-19 (PRONCOVID): a prospective cohort study. Lancet Respir Med 2020;8:765-74.

18. Caputo ND, Strayer RJ, Levitan R. Early self-proning in awake, non-intubated patients in the emergency department: A single ED's experience during the COVID-19 pandemic. Acad Emerg Med 2020;27:375-8.

19. Retucci M, Aliberti S, Ceruti C, et al. Prone and lateral positioning in spontaneously breathing patients with COVID-19 pneumonia undergoing noninvasive helmet CPAP treatment. Chest 2020;158:2431-5.

20. Tu G-W, Liao Y-X, Li Q-Y, et al. Prone positioning in highflow nasal cannula for COVID-19 patients with severe hypoxemia: a pilot study. Ann Transl Med 2020;8:598.

21. Golestani-Eraghi M, Mahmoodpoor A. Early application of prone position for management of Covid-19 patients. J Clin Anesth 2020;66:109917.

22. Ng Z, Tay WC, Ho CHB. Awake prone positioning for nonintubated oxygen dependent COVID-19 pneumonia patients. Eur Respir J 2020;56:2001198.

23. Moghadam VD, Shafiee H, Ghorbani M, Heidarifar R. [Prone positioning in management of COVID-19 hospitalized patients].[Article in ortuguese]. Rev Bras Anestesiol 2020;70:188-90.

24. Huang M, Yang Y, Shang F, et al. Clinical characteristics and predictors of disease progression in severe patients with COVID-19 Infection in Jiangsu Province, China: A descriptive study. Am J Med Sci 2020;360:120-8.

25. Ripoll-Gallardo A, Grillenzoni L, Bollon J, et al. Prone positioning in non-intubated patients with COVID-19 outside the intensive care unit: More evidence needed. Disaster Med Public Health Prep 2020;14:e22-4.

26. Despres C, Brunin Y, Berthier F, et al. Prone positioning combined with high-flow nasal or conventional oxygen therapy in severe Covid-19 patients. Crit Care 2020;24:256.

27. Thompson AE, Ranard BL, Wei Y, Jelic S. Prone positioning 
in awake, nonintubated patients with COVID-19 hypoxemic respiratory failure. JAMA Intern Med 2020;180:1537-9.

28. Xu Q, Wang T, Qin X, et al. Early awake prone position combined with high-flow nasal oxygen therapy in severe COVID19: a case series. Crit Care 2020;24:250.

29. Damarla M, Zaeh S, Niedermeyer S, et al. Prone positioning of non-intubated patients with COVID-19. Am J Respir Crit Care Med 2020;202:604-6.

30. Quah P, Li A, Phua J. Mortality rates of patients with COVID19 in the intensive care unit: a systematic review of the emerging literature. Crit Care 2020;24:285.

31. Rubenfeld GD. Confronting the frustrations of negative clinical trials in acute respiratory distress syndrome. Ann Am Thorac Soc 2015;12:S58-63.
32. Delclaux C, L'Her E, Alberti C, et al. Treatment of acute hypoxemic nonhypercapnic respiratory insufficiency with continuous positive airway pressure delivered by a face mask: A randomized controlled trial. JAMA 2000;284:2352-60.

33. Mittal S, Pb S, Madan K, Mohan A, et al. Awake proning in COVID-19 - does CPAP make a difference? Adv Respir Med 2021;89:82.

34. Sryma PB, Mittal S, Madan K, et al. Reinventing the wheel in ARDS: Awake proning in COVID-19. Arch Bronconeumol 2020;56:747-9.

35. Pierce-Williams RAM, Burd J, Felder L, et al. Clinical course of severe and critical COVID-19 in hospitalized pregnancies: a US cohort study. Am J Obstet Gynecol MFM 2020;2: 100134. 